\title{
Bodily, emotional, and public sphere at the time of COVID-19. An investigation on concrete and abstract concepts
}

\author{
Claudia Mazzuca $^{1}$ (I) Ilenia Falcinelli $^{1} \cdot$ Arthur-Henri Michalland $^{2} \cdot$ Luca Tummolini $^{3} \cdot$ Anna M. Borghi $^{1,3}$
}

Received: 23 June 2021 / Accepted: 12 December 2021 / Published online: 24 January 2022

(c) The Author(s), under exclusive licence to Springer-Verlag GmbH Germany, part of Springer Nature 2021

\begin{abstract}
The outbreak of Covid-19 pandemics has dramatically affected people's lives. Among newly established practices, it has likely enriched our conceptual representations with new components. We tested this by asking Italian participants during the first lockdown to rate a set of diverse words on several crucial dimensions. We found concepts are organized along a main axis opposing internal and external grounding, with fine-grained distinctions within the two categories underlining the role of emotions. We also show through a comparison with existing data that Covid-19 impacted the organization of conceptual representations. For instance, subclasses of abstract concepts that are usually distinct converge into a unitary group, characterized by emotions and internal grounding. Additionally, we found institutional and Covid-19 related concepts, for which participants felt more the need for others to understand the meaning, clustered together. Our results show that the spread of Covid-19 has simultaneously changed our lives and shaped our conceptual representations.
\end{abstract}

\section{Introduction}

Concepts are the building blocks of our knowledge, the glue that ties our past, present, and future together (Murphy, 2002). But concepts are also flexible, they may be stretched or shrunk as a consequence of changes in the physical or social environment. Some authors have underlined that concepts are dynamic and flexible (Barsalou, 1993; Connell \& Lynott, 2014). Still, research has mostly focused on stable and constant aspects of conceptual representations due to the difficulty of investigating conceptual flexibility in everyday life. The spread of Covid-19 represents a unique occasion that allows us to investigate how an unexpected and disruptive event on such a large scale has changed our way of conceiving our bodies, emotions, as well as our personal and social lives. In this work, we explore whether and how the

Claudia Mazzuca

mazzuca.claudia@gmail.com

Anna M. Borghi

anna.borghi@uniroma1.it

1 Department of Dynamic, Clinical Psychology and Health, Sapienza University of Rome, Rome, Italy

2 University of Montpellier, LIFAM, Montpellier, France

3 Institute of Cognitive Sciences and Technologies, Italian National Research Council, Rome, Italy way we represent concepts reflects the changes that occurred in society as a consequence of the Covid-19 outbreak.

To investigate the impact of the spread of Covid-19 in different areas, we designed a study in which we asked participants to rate 96 words according to various dimensions. Most of the words were taken from previous Italian databases, in which either abstract (Villani et al., 2019) or both concrete and abstract concepts were evaluated (Barca et al., 2002; Della Rosa et al., 2010). To investigate the spheres in which the pandemics could have impacted people's life, we selected words that belong to the domains of public, personal, emotional, and bodily spheres. Notably, some of the selected words were more concrete (e.g., body, school), some more abstract (e.g., destiny, altruism). The distinction of concepts into concrete and abstract, although highly simplified (Barsalou et al., 2018), reflects the fact that some concepts (e.g., stomach) refer to concrete, single, and clearly bounded referents, activate sensorial modalities to a larger extent, and have many common properties (high-dimensional categories: Langland-Hassan et al., 2021; Lupyan \& Mirman, 2013), while other concepts, i.e., more abstract ones (e.g., future), are more detached from sensorial experience, collect rather heterogeneous members, and generally refer to complex events and situations (Borghi \& Binkofski, 2014). We hypothesized that abstract concepts, being more relational and less linked to the correlational structure of the outside environment than concrete ones (Gentner \& 
Asmuth, 2019; Malt \& Wolff, 2010; Thompson et al., 2020), are more flexible and more influenced by contextual factors than concrete ones.

To test this hypothesis, we chose words that pertained to different semantic domains. First, we considered words related to diseases, differing in severity (from flu to tumor, including Covid-19), and epidemiological words (e.g., exponential growth, social distancing), which can be considered less familiar words for lay people-since they became more frequently used only during the pandemic spread. We also selected concepts related to potentially risky collective situations (e.g., crowd, demonstration), and concepts of locations that might have become more salient, either because of their risk or because of people's confinement (e.g., airport, home). For the bodily sphere, we selected mostly concepts that might have become more salient due to the pandemic (e.g., mouth, face, breath). For the emotional sphere, we selected abstract words referring to simple and complex emotions, both positive and negative (e.g., fear, anxiety, love, trust), and words referring to spiritual life and religion, both positive and negative (e.g., religion, salvation, destiny, death) (Villani et al., 2019). For the private sphere, we selected kinship words (e.g., grandparent, sister), and to investigate changes in the public sphere, we considered institutional concepts (e.g., state, nation), and social concepts referring to professions that have become more salient during the pandemics (e.g., prime minister, journalist). We also included nations, the representation of which might have changed due to Covid-19 (e.g., China, France).

We used an online rating method in which we asked participants to rate words according to many dimensions. First, we selected three dimensions classically used to determine conceptual abstractness. Participants evaluated how much the words were abstract/concrete (Concreteness-Abstractness), how likely they were to evoke images (Imageability, Paivio, 1990), and to what extent it was easy to think of possible contexts associated with them (Contextual Availability, McFalls \& Schwanenflugel, 2002). Generally, people evaluate more concrete concepts as more imageable and more associated with a given context (McFalls \& Schwanenflugel, 2002; Paivio, 1990). Then, we chose four dimensions that have been recently tested on abstract concepts. Three pertained to the body and aimed at determining whether and to what extent the words evoke bodily interactions (Body Object Interaction, BOI, Tillotson et al., 2008), signals from the inner body (Interoception, Connell et al., 2018; Villani et al., 2021), and emotions (Emotionality, Kousta et al., 2011). The last one addressed to what extent participants had the feeling to need others to understand the word meaning (Social Metacognition, Borghi et al., 2018, 2019). Previous studies on abstract concepts (Villani et al., 2019; 2021) revealed that higher BOI scores generally characterize more concrete concepts among abstract concepts, and that Emotionality and Interoception are more associated with emotional and mental states abstract concepts. Furthermore, the degree of Social Metacognition increases with the increase of abstractness - the more words are abstract, the more we feel the need of others.

Finally, we added some novel dimensions, addressing the extent to which people felt confident about conceptual meaning (Confidence), the extent to which they thought experts understand the concept (Confidence in Experts), and the extent to which they perceived the conceptual referent as near or far from them (Distance). We introduced Confidence and Confidence in Experts to refine the measurement of Social Metacognition. We were interested in understanding whether we need others more for concepts for which we feel less confident. Furthermore, we intended to assess if the confidence in experts' knowledge could fill this gap and reduce the perceived uncertainty (Rabb et al., 2019). We reasoned that questions to a possible community of knowledge may be particularly relevant for abstract, scientific concepts (e.g., exponential growth), and epidemiological concepts (e.g., disease), and would help to address contextual changes due to the pandemic. Finally, we hypothesized that participants would perceive abstract concepts as more distant because of their difficulty, together with concepts referring to locations and populations evoking worrying scenarios in the initial phase of the pandemics (e.g., airport, China).

For a subset of these words, we have previous evaluations of other databases. Even if the sample of words participants evaluated was different overall, comparing ratings obtained during the pandemics to those already available from previous norming studies allows us to determine whether and to what extent the spread of the pandemics affected conceptual representation on some critical dimensions.

\section{Method}

\section{Participants}

A total of 36 Italian participants took part in the study in a window of time between April, 2nd and May, 9th 2020i.e., in the initial phase of the first Italian lockdown. The questionnaire was implemented in Qualtrics. Participants were contacted via an anonymous link either by posting the questionnaire on social networks (Facebook, Twitter) or spreading the questionnaire through the research team's extended network of acquaintances. Originally we contacted 106 people, but 70 of them (66.04\% of the sample) did not complete the questionnaire, likely because of its length. The final sample is therefore composed of 36 participants ( 23 females, $M$ age $=35.87 ; \mathrm{SD}=13.04 ; 13$ males, $M$ age $=36.54 ; \mathrm{SD}=12.60)$. 
Participants showed middle levels of trait anxiety $(M=2.26$; range 1-4; $\mathrm{SD}=0.66)$ tested with the GAD-7 scale (Spitzer et al., 2006), middle levels of empathy $(M=3.76$; range 2-5; $\mathrm{SD}=0.65)$ tested with the IRI scale (Davis, 1980), and middle-high levels of interoceptive awareness $(M=4.33$; range $2.5-6 ; \mathrm{SD}=0.94)$ tested with the MAIA scale (Mehling et al., 2012). Their perception of the risk of contracting Covid-19 was average (score 3.78; $\mathrm{SD}=1.33$, tested with a continuous scale from 1 to 7 ), and the frequency with which they assessed information and news about Covid-19 was middle-high $(M=3.97 ; \mathrm{SD}=0.84$, tested with a scale from 1 to 5).

All the other socio-demographic information collected are reported in Appendix A (see Supplementary Materials).

\section{Materials}

Participants took part in an online survey. In the first part of the survey, participants completed the rating task: they were asked to evaluate a list of words $(N=96)$ according to specific dimensions, using a scale from 1 to 7 .

We tested 10 dimensions, including Concreteness $\sim$ Abstractness, Imageability, Context Availability, Body-Object-Interaction, Social Metacognition, Interoception, and Emotionality (Della Rosa et al., 2010; Villani et al., 2019). To these, we added three new dimensions: Perceived Distance from the concept, Confidence in Experts, and Confidence (see Appendix B, Supplementary Materials).

The 96 target words pertained to 11 categories, which were: Diseases, Epidemiological Concepts, PhilosophicalReligious Concepts, Emotional Concepts, Concepts referred to Body and Senses, Social Professions, Social and Collective Concepts, Concepts referred to Family, Institutional Concepts, Places-Means of Transport, and Nations (see Appendix C, Supplementary Materials).

The ten dimensions were randomly presented within participants, and the 96 target words were randomly presented within each dimension.

In the second part of the survey, participants were asked to complete four scales: the Interpersonal Reactivity Index (IRI) (Davis, 1980), testing their general empathy; the Multidimensional Assessment of Interoceptive Awareness (MAIA) (Mehling et al., 2012), investigating their interoceptive awareness; the Stereotype Content Model (SCM) (Cuddy et al., 2009) that investigates the content of the stereotypes endorsed by individuals towards specific social groups; and the Generalised Anxiety Disorder-7 (GAD-7) (Spitzer et al., 2006) aimed at measuring the severity of Generalized Anxiety Disorder.

In the third part of the survey, we collected socio-demographic information. We asked participants to report their age, birth sex, level of education, profession, birth nation, city and region of provenience, current health condition, current way of living (confined or not confined to the house), frequency with which they received information and news about Covid-19, and personal perceived risk to contract Covid-19 (see Appendix A, Supplementary Materials).

\section{Procedure}

Ethics permission was granted by the Ethics Committee of the Department of Dynamic, Clinical Psychology and Health, Sapienza University of Rome (Prot. n. 000275$23 / 03 / 2020$ ). Before completing the survey, participants were informed of the general purpose of the study and provided informed consent. Once participants agreed to take part in the study, they were presented with the rating task and asked to evaluate target words on ten dimensions, using a continuous scale from 1 to 7 . They were encouraged to respond as carefully and quickly as possible, without spending too much time thinking about every single word. Participants gave their responses into separate boxes for each target word contained per dimension. All data, scripts, and analyses are available at https://osf.io/h8t59/.

\section{Results}

To assess meaningful relations among our targeted dimensions, we computed Spearman's rho coefficients for all possible pairs of dimensions in our matrix using 'Hmisc' R's package (Harrell, 2020). Figure 1 presents a correlogram implemented through 'corrplot' R's package (Wei \& Simko, $2017)$ showing the outcome of the correlations. In the correlogram, only significant correlations $(p>0.05)$ are reported. $p$-values have been adjusted for multiple comparisons using Bonferroni's corrections. Below, we discuss the most meaningful relations.

We found that more abstract concepts received lower scores of Imageability, $r_{\mathrm{s}}=-0.98, p<0.001$, and Contextual Availability, $r_{\mathrm{s}}=-0.89, p=0.049$, in line with widely documented findings on abstractness (e.g., Paivio, 1986; Schwanenflugel \& Shoben, 1983), and of Body-ObjectInteraction (BOI), $r_{\mathrm{s}}=-0.94, p=0.005$, in keeping with recent findings (Villani et al., 2019).

Concepts that were rated as more distant were also rated as less emotional, $r_{\mathrm{s}}=-0.96, p=0.001$, and less related to interoception, $r_{\mathrm{s}}=-0.94, p=0.005$. Concepts for which participants judged the need of others to be crucial for understanding the meaning (Social Metacognition; METSOC) received also lower scores of BOI, $r_{\mathrm{s}}=-0.93, p=0.010$ and Confidence in the mastering of meaning, $r_{\mathrm{s}}=-0.99$, $p<0.001$. Interestingly, these results confirm the link between abstractness and social metacognition, suggested by recent understandings of abstract knowledge (e.g., Borghi et al., 2021; Fini \& Borghi, 2019; Shea, 2018; Villani et al., 


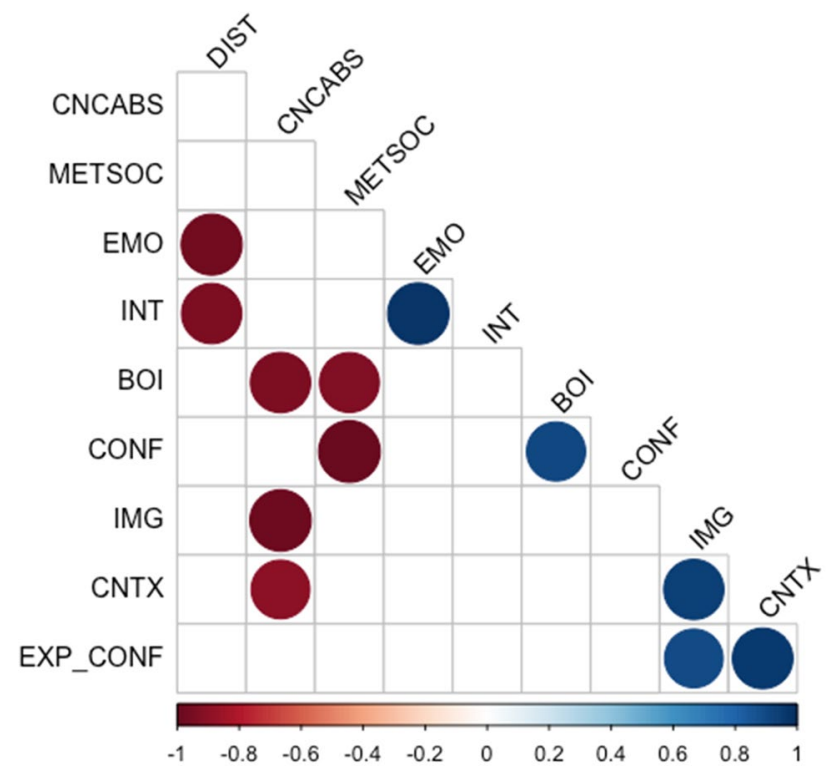

Fig. 1 Correlogram of significant relations among the dimensions. Positive correlations are reported in blue, whereas negative correlations are reported in red. Legend of labels: CNCABSConcreteness $\sim$ Abstracntess, METSOCSocial Metacognition, EMOEmotionality, INTInteroception, BOIbody object interaction, CONFConfidence, IMG imageability, CNTX contextual availability, EXP_CONFConfidence in Experts, DISTPerceived Distance

2019). In keeping with this idea, social interaction would be central for understanding and consolidating the meaning of abstract concepts.

We found a positive correlation between Emotionality and Interoception, $r_{\mathrm{s}}=0.98, p<0.001$. It is perhaps worth noting that, contrary to previous research (e.g., Kousta et al., 2011; Lund et al., 2019; Ponari et al., 2018), we found no significant correlation between Abstractness and Emotionality $(p>0.05)$. Instead, these results align well with proposals suggesting that emotional concepts can be considered as a category differing from both concrete and abstract concepts (e.g., Altarriba et al., 1999; Barca et al., 2017, 2020). We also found a positive correlation between Body-Object-Interaction and Confidence, $r_{\mathrm{s}}=0.90, p=0.031$, and Imageability and Confidence in Experts' Knowledge, $r_{\mathrm{s}}=0.89, p=0.049$. The correlation suggests that confidence in ourselves and in experts increases when items are perceived as under our (bodily) control and as easily imageable, two determinants of concreteness. Finally, we also found a positive correlation between Imageability and Contextual Availability, $r_{\mathrm{s}}=0.94$, $p=0.002$, in line with previous research (e.g., Villani et al., 2019).

To further explore our data and to identify underlying components explaining their structure, we performed a Principal Component Analysis (PCA, Jollife, 2010) using 'tidymodels' (Kuhn \& Wickham, 2020) and 'factoextra' (Kassambara \& Mundt, 2019) R's packages. PCA is a multivariate analysis technique that allows extracting a new set of features from an existing set of variables, explaining the variance in the dataset. Before applying PCA, all the variables (i.e., the average rating score per each dimension) were centered and standardized.

We focus on components that weigh more of I.3I, which can be interpreted as medium effect size (Cohen, 1988; see also Villani et al., 2019). We found that, among the ten components extracted by the PCA, component 1 (PC1) explained the majority of variance $(0.51)$, followed by component 2 (0.28). Figure 2 presents the contribution of each dimension on PC1 and PC2 and their negative and positive values.

Here we focus on the most relevant dimensions (for a complete overview of the contribution of each dimension on both components, see Appendix D, Supplementary Materials). On the first component (PC1), we found that Imageability ( -0.42$)$, Body-Object-Interaction $(-0.39)$, Confidence in understanding the meaning $(-0.36)$, Contextual Availability ( -0.35$)$, and Confidence in experts' knowledge ( -0.33 ) are opposed to Abstractness (0.40), and Social Metacognition (0.31). This is in line with the pattern of associations revealed by correlations and suggests that Component 1 represents the opposition between abstractness and concreteness. Compared to previous studies, the opposition between abstractness and concreteness is enriched by the information on confidence. The more abstract the words are, the less confident we are both in our own and in the experts' knowledge. At the same time, with more abstract words we need more others to understand their meaning, confirming results by Villani et al. (2019) and showing that social metacognition is an important dimension that characterizes abstractness.

On the second component (PC2), Interoception (0.52) and Emotionality (0.52) are opposed to Perceived Distance $(-0.45)$ and Confidence in Experts' knowledge $(-0.26)$, once again replicating the trend of the correlations. This component mainly opposes concepts that are highly related to emotions and interoception to those perceived as more distant and more understood by experts, somehow displaying a "near $\sim$ far" opposition. The relation between distance and better comprehension of experts might seem counterintuitive, but it can be better understood if we consider that distant objects are opposed to inner, emotional aspects, more related to the personal dimension.

\section{How are words organized in the PCA semantic space?}

Once the most salient components of the PCA have been identified, we can analyse the organization of the target words in the resulting semantic space. Figure 3 presents the distribution of target words on the bidimensional 
Fig. 2 Contribution of each dimension on $\mathrm{PC} 1$ and $\mathrm{PC} 2$, and their positive and negative values

Fig. 3 Distribution of target words on each component. The distance between dimensions and the origin roughly measures the quality of the dimensions on the factor map. Dimensions that are distant from the origin are well represented on the factor map. Target words that are on the same side of a dimension have high values for that dimension, and vice versa
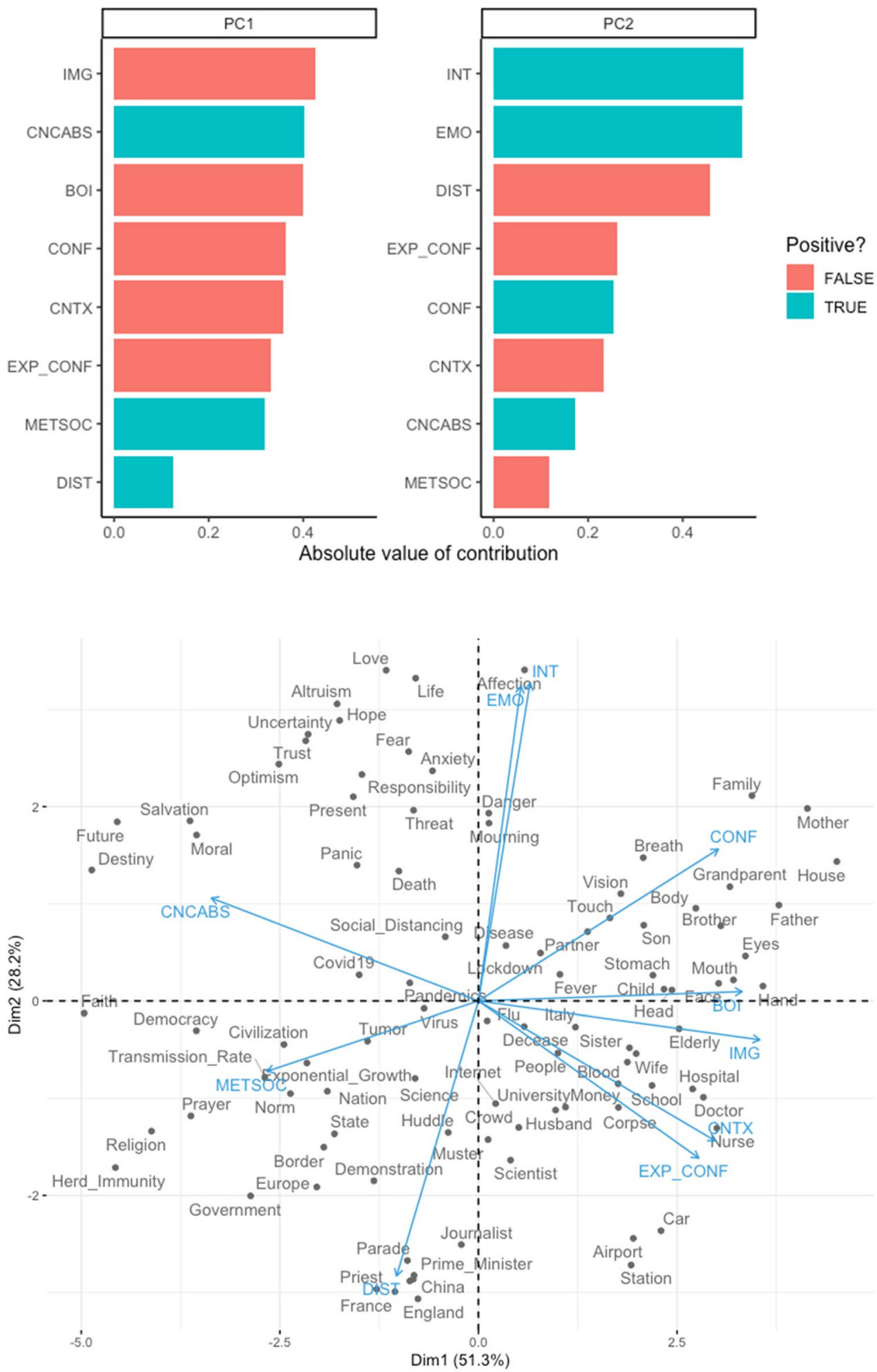

space resulting from PCA, along with the contribution of dimensions.

The first component (Dimension 1) accounts for most of the variability in the dataset. As the plot shows, on Dimension 1 highly imageable words (e.g., elderly, hospital) are distributed together with words that are easily available in contexts (e.g., car, school), highly related to bodily experience (e.g., eyes, hand, mouth, head, face), and on the meaning of which participants felt confident (e.g., family, mother, house). These words are opposed in the leftmost part of the 
plot to words that received higher scores of abstractness (e.g., future, destiny, moral), to words for which the need of others to understand the meaning was considered to be important (e.g., norm, prayer, herd immunity, democracy), and to words that were perceived as more distant (e.g., England, demonstration, journalist).

In the second component (Dimension 2) we find on the one hand highly emotional words, and words related to interoceptive feelings (e.g., affect, life, fear, anxiety, hope, love, faith, but also breathe, sight, touch, illness), which are opposed to words that are perceived as distant (e.g., crowd, government, Europe, science, Britain, demonstration) and that were judged to be well understood by experts (e.g., scientist, physician, corpse, airport).

To better interpret our data, we performed Hierarchical Clustering on the results of the Principal Component Analysis using "FactoMineR" R's package (Lê et al., 2008). Silhouette's Index (see Chouikhi et al., 2015) indicated three as the best number of clusters for our data $(\mathrm{SI}=0.3)$. We identified relevant clusters based on their Euclidean distance on the dimensions of ratings, using Ward's clustering algorithm (see Harpaintner et al., 2018; Mazzuca et al., 2020 for similar methods), an agglomerative clustering algorithm that minimizes the total within clusters variance. Figure 4 presents the distribution of words in each cluster and their distribution in the bidimensional space of the PCA.
The three clusters are differentially characterized by the dimensions, $F(18,930)=58.53, p<0.001$.

Specifically, words included in Cluster 1 (green) have higher scores of Emotionality ( $M=5.26$; Overall $M=4.25$ ), Abstractness $(M=4.34$; Overall $M=3.17)$, Interoception $(M=4.96$; Overall $M=3.97)$ in comparison with the overall tendency of the data. In addition, they have lower scores of Contextual Availability $(M=3.64$; Overall $M=4.55)$. Words included in Cluster 2 (orange) are instead mainly characterized by higher scores of perceived Distance $(M=4.13$; Overall $M=3.57)$ and lower scores in Confidence $(M=4.66$; Overall $M=5.09$ ). Finally, words that are grouped together in Cluster 3 (blue) have higher scores of BOI $(M=5.10$; Overall $M=4.44)$, Imageability $(M=5.40$; Overall $M=4.79)$, and lower scores of Abstractness $(M=2.25$; Overall $M=3.17$ ). A visual inspection of the words composing the three clusters suggests that Cluster 1 contains mostly emotional and abstract words; Cluster 2 seems to be composed of institutional, public concepts that are specifically related to the Covid pandemics; Cluster 3 instead features mainly highly concrete, bodily related words the representation of which might have been also influenced by the pandemics. This pattern of results aligns well with the results of the PCA. Figure 5 presents the mean contribution of each cluster on the two main components of the PCA. To make the interpretation of results easier, we have named the
Fig. 4 Distribution of target words on each component, and their distribution in each cluster

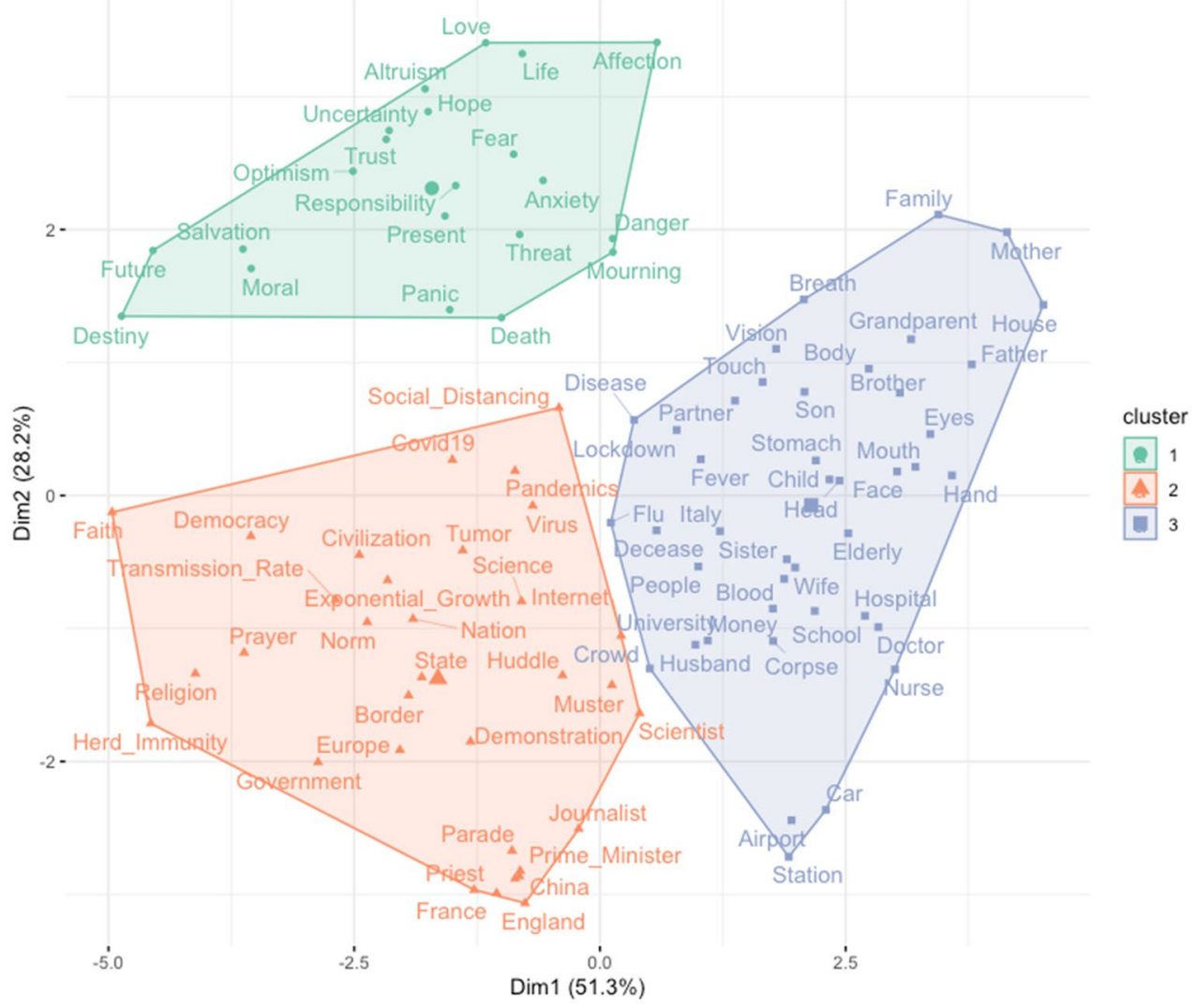




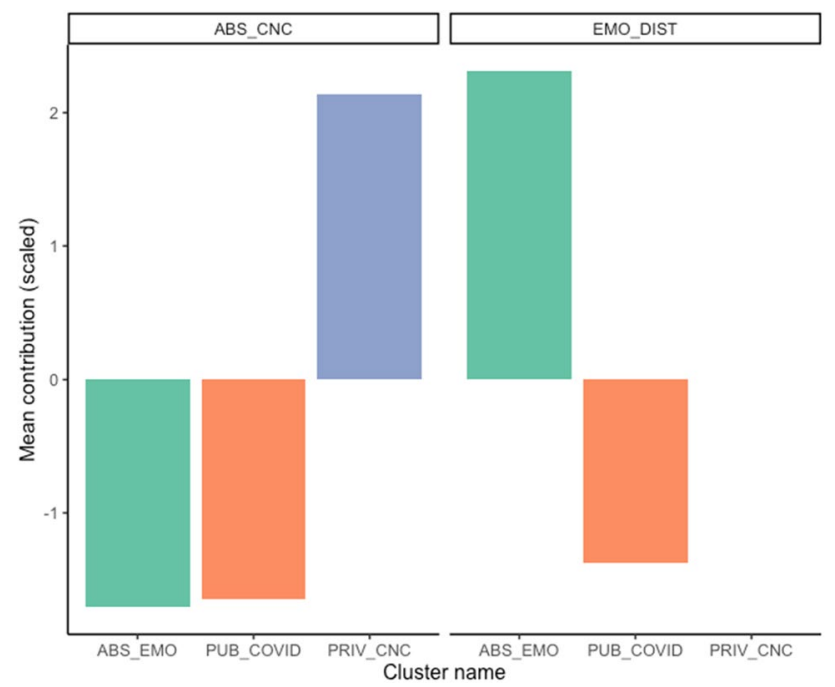

Fig. 5 Mean contribution (scaled) of each cluster per each component of the PCA. Colors of clusters match those reported in Fig. 4

two components in line with the dimensions that better characterized them, so that PC1 is now ABS_CNC (representing the opposition between abstractness and concreteness), and PC2 is EMO_DIST (representing the opposition between emotionality and perceived distance). We did the same with the three clusters so that Cluster 1 is now ABS_EMO (abstract and emotional), Cluster 2 is PUB_COVID (public, institutional aspects mediated by the Covid pandemics), and Cluster 3 is PRIV_CNC (concrete, bodily salient, private aspects).

The mean contribution of clusters to the components of the PCA suggests that Component 1 (ABS_CNC) is generally characterized by the opposition between words pertaining to Cluster 3 (PRIV_CNC), which have the higher contribution to the dimension, and words in Cluster 1 (ABS_EMO). Component 2 (EMO_DIST) is instead characterized by the opposition between words clustered in Cluster 1 (ABS_EMO), which have the higher contribution, and words in Cluster 2 (PUB_COVID). It is worth noting that words that compose the PUB_COVID cluster seem to have comparable importance in both dimensions. This could be explained by the fact that they were rated as relatively abstract words, for which other people are determinant for understanding the meaning, but were also perceived as somehow distant.

These are interesting results for a variety of reasons. First, we found a cluster including only abstract words and of two different kinds. Words that in Villani et al. (2019) belonged to two separate clusters, i.e., emotional-inner states words and philosophical-religious ones, here cluster together, whether positive or negative in valence (e.g., salvation, hope, fear, love). Whether the assimilation of these two kinds of abstract words is a consequence of experiencing an unusual and highly emotional situation, such as the spread of Covid-19, or whether it depends on the different sample of presented words, are both possible explanations that should be tested in future studies. According to some accounts (e.g., Kousta et al., 2011), we might expect that highly abstract and emotional terms would be grouped together. However, our data indicate that we might expect the opposite pattern: in fact, even though we had mostly Covid-related highly abstract words (e.g., exponential growth), we found these words included together in a cluster mainly characterized by the dimensions of Social Metacognition, perceived Distance, and low Confidence.

Second, although we cannot determine whether and to what extent the spread of Covid-19 has influenced the formed clusters, some cues suggest that it plays a role for both Cluster 2 and 3. Cluster 2 includes words related to pandemics and the public sphere. Together with the word Covid-19, we find scientific words (e.g., exponential growth, herd immunity, transmission rate, science), spaces where the contagion can spread (e.g., parade, demonstration), or locations associated with it (e.g., China, England), together with institutional words related to institutional spaces and figures (e.g., state, norm, prime minister, journalist). Cluster 3 includes mostly concrete words related to the private sphere, and perhaps surprisingly, the word lockdown. It then includes words referring to bodily parts and family members, to diseases (disease, $f u$ ) and death (corpse), to the hospital (e.g., nurse, hospital), to the nation where people were confined (Italy). Notably, the words school and university are included in this cluster and not in Cluster 2, either because during the lockdown lessons were only online or because people perceive school and university as part of everyday life. It is perhaps worth noting that other countries are clustered with more "abstract" Covid-19 terms (pandemic, Covid-19, exponential growth), whereas Italy (i.e., participants' home country) clustered with more perceptible, maybe more bodily consequences of covid (lockdown, disease, fever).

Finally, to assess whether the Covid pandemics have impacted our conceptual representations, we retrieved rating scores from an Italian database of abstract words (Villani et al., 2019), which features most of the dimensions included in our dataset. Importantly, all the words in the Villani's database are associated with one of three conceptual categories: "emotional-inner"; "philosophicalspiritual"; "physical-spatial"; "self-sociality". We found 29 words out of 96 (see Supplementary Materials), and we fitted a linear mixed model using 'afex' R's package (Singmann et al., 2021). We modeled our data fitting rating scores as the dependent measure, Database (our database vs Villani), Dimension, and Category as fixed factors, and Target Word as a random factor. We found a significant main effect of Dimension, $F(6,325)=58.15, p<0.001$, 
as well as a significant main effect of Category, $F(3$, $25)=7.96, p<0.001$. We also found a significant interaction between Database and Dimension, $F(6,325)=8.19$, $p<0.001$, and between Dimension and Category, $F(18$, $325)=12.88, p<0.001$. Importantly, the three-way interaction between Database, Dimension, and Category was also significant, $F(18,325)=1.72, p=0.035$. To further explore the direction of this interaction, we computed post-hoc comparisons using 'emmeans' (Lenth, 2020) R's package and adjusted $p$-values using Tukey's correction. Within the four categories, we found that only "emotional-inner" and "philosophical-spiritual" concepts were rated differently across the two databases. Specifically, for ratings of Contextual Availability "emotional-inner" concepts received higher scores in Villani's database, $t(325)=9.042, p<0.001$, as well as "philosophical-spiritual" concepts, $t(325)=7.125, p<0.001$. For Imageability, "emotional-inner" concepts received higher scores in Villani's database, $t(325)=5.232, p<0.001$, and the same is true for "philosophical-spiritual" concepts, $t(325)=4.162$, $p=0.001$. For Social Metacognition, "emotional-inner" concepts received lower scores in Villani's database, $t(325)=-3.187, p=0.033$, as did "philosophical-spiritual" concepts, $t(325)=-3.789, p=0.004$. Figure 6 presents the trend of the interaction.

Even if we cannot exclude that these differences are due to the different participants' pool, to the fact that Villani et al. had participants evaluating only abstract concepts, or to the spread of the epidemics, our results on this subset of words offer interesting ideas for interpretation. Indeed, the difference pertains to concepts referring to the emotional and spiritual sphere and not other concepts. Within these two categories, we found a decrease in Imageability, and Contextual Availability, likely due to the different word samples. In fact, in our database, these abstract concepts are compared with concrete ones, possibly leading to an overall decrease in scores on dimensions traditionally associated with concreteness. However, it is worth noting that changes occur with Imageability and Contextual Availability, and not with other dimensions, such as abstractness $\sim$ concreteness.

The most interesting result concerns the difference in Social Metacognition, indicating that the current situation has enhanced participants' need to rely on others to

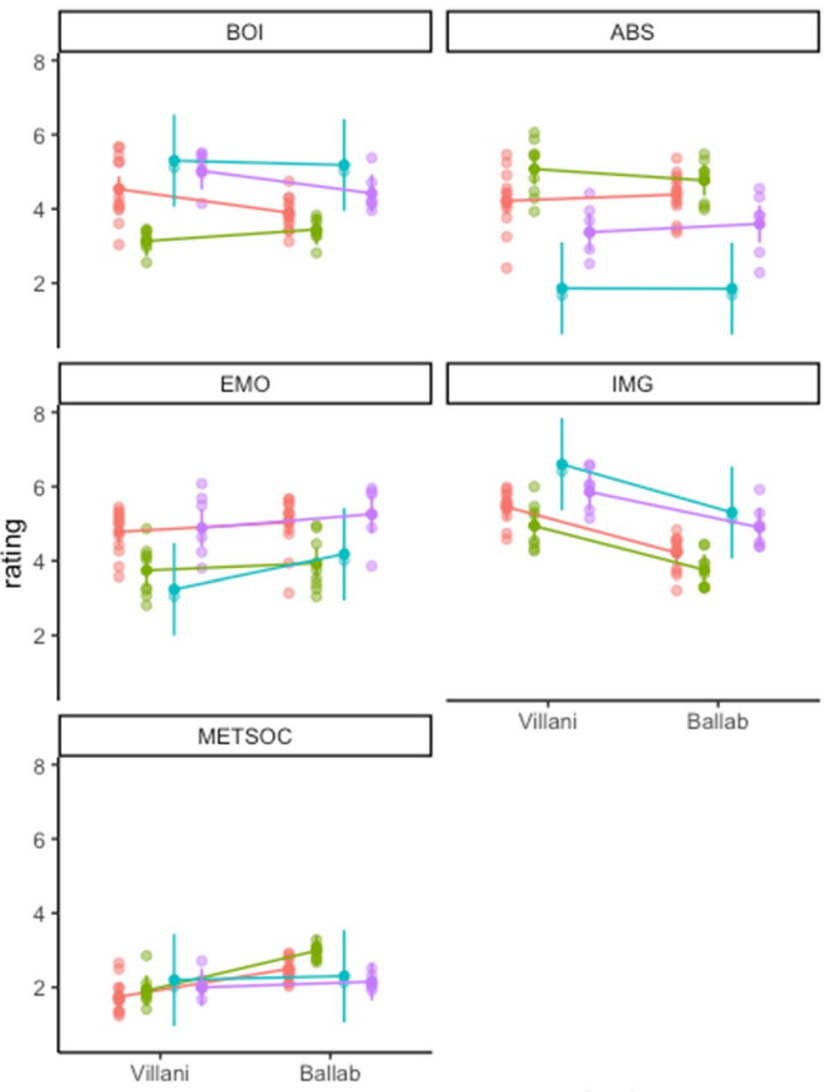

database
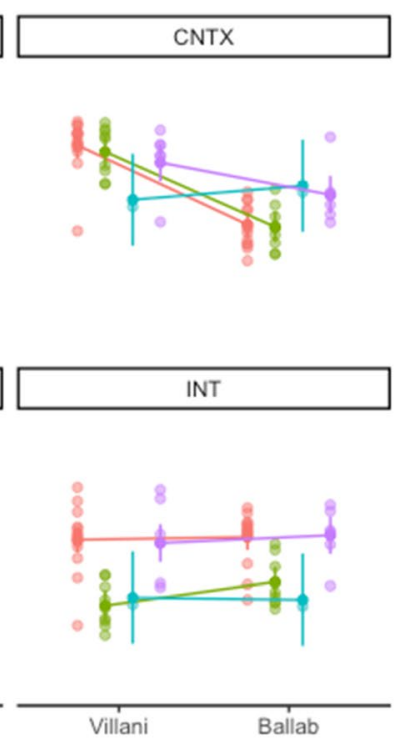

Category_Villani

- emotion-inner

$\multimap$ philosop-spiritual

- physical-spatial

$\rightarrow$ self-sociality

Fig. 6 Estimated marginal means for target words $(n=29)$ by dimension and categories of Villani et al.'s (2019) database of abstract words in both Villani's and our (Ballab) ratings 
understand the meaning of both "emotional-inner" and "philosophical-spiritual" concepts.

\section{Discussion}

In this study, we explored whether and how the spreading of Covid-19 affected Italian participants' conceptual representations. To this extent, we asked an Italian sample of participants during the first national lockdown (March 2020) to rate a set of target words on a number of dimensions usually employed to test conceptual concreteness (e.g., Imageability, Abstractness, Contextual Availability, Body-Object-Interaction, Emotionality), along with less traditional measures (e.g., Confidence, Social Metacognition, Confidence in Experts, Perceived Distance). Our results can be interpreted in light of two main interrelated research strands. The first is related to the literature on conceptual representation, and the dimensions that underlie the abstractness of concepts; the second tackles how a disruptive event such as Covid-19 has impacted our concepts. We will address them separately.

\section{Concepts and their abstractness}

Our results directly speak to the literature investigating conceptual representations and specifically to the distinction between abstract and concrete concepts. In fact, although we did not select our target concepts explicitly controlling for abstractness, the results of the Principal Component Analysis showed that the abstract $\sim$ concrete opposition accounted for most of the variance in the dataset. This result seems to be in contrast with recent perspectives on conceptual knowledge (e.g., Barsalou et al., 2018; Borghi et al., 2019), which are questioning the traditional dichotomy opposing abstract to concrete concepts. However, these approaches are also stressing the multidimensionality of abstract concepts (Borghi, Fini, et al., 2021; Borghi et al., 2021) suggesting that other underlying dimensions might account for the representation of abstract meanings. In keeping with that, along with classic dimensions explaining concreteness and abstractness (i.e., Contextual Availability, Imageability), we found this distinction is further enriched by the association between abstractness and Social Metacognition, i.e., the tendency to rely on others to understand conceptual meaning. Some research strands propose that this dimension might be critical for abstract concepts' acquisition and mastering (e.g., Borghi et al., 2018, 2021; Mazzuca et al., 2021a). In line with this proposal, abstract conceptual representations might be established and reinforced also through a dynamic loop constituted by the conscious or unconscious assessment of the inadequacy of our conceptual knowledge, and the consequent social interactions aimed at integrating and enriching it. This might be especially relevant for specific kinds of abstract concepts, such as those related to scientific or more complex entities (e.g., herd immunity). Importantly, in our study information on confidence also enriches how abstract and concrete concepts are conceived, showing that confidence both in our own knowledge and in experts' knowledge decreases with more abstract concepts. So, while positing a clear-cut distinction between abstract and concrete concepts might be a simplification (Barsalou et al., 2018), our results show that the abstract concrete distinction, even if nuanced, seems to be an important underlying factor (see also Dove, 2018). Importantly, the abstract concrete dimension (PC1) did not entirely overlap with emotionality. Instead, we found that the opposition between Emotionality and Perceived Distance distinctively characterized the second component of our PCA, marking the distinction between concepts that are perceived as internally grounded and concepts that are perceived as distant. This does not imply that emotionality does not count for abstract concepts. Indeed, the analysis of Cluster 1 indicates that, together with interoception, it plays a major role, but particularly for emotional-inner and philosophical-religious concepts. This result suggests that theories claiming that emotional information is more crucial for abstract than concrete concepts (e.g., Kousta et al., 2011; Lund et al., 2019) capture an important dimension of abstract concepts. However, the relevance of emotionality and interoception (Connell, 2019) is particularly prominent for a subset of abstract concepts, i.e., emotional and religious ones (see Villani et al., 2021).

\section{Effects of Covid-19 on concepts}

Our results offer hints to understand the influence of the SARS-CoV-2 spread on conceptual representations. They provide clear evidence that participants' conceptual representations were at least partly modulated by the emergency context caused by the pandemics (for related findings see Mazzuca et al., 2021b).

First, in the cluster analysis, we observe a more tight relationship between emotional and inner states concepts (e.g., fear, love) and philosophical, spiritual ones (e.g., destiny, salvation) compared with previous studies on abstract concepts. While these two sub-classes of concepts were found to be differentially represented in a previous Italian norming study (Villani et al., 2019), here they both fall in the same cluster. Although these results are based on a smaller set of words, we also found that emotional-inner and philosophical-spiritual concepts were the only two sub-categories that varied in the two databases' ratings. Particularly relevant is the increase in Social Metacognition scores. In our dataset, the need to rely on others to understand the meaning of these two kinds of concepts is more substantial, consistently with the hypothesis that the pandemics might have increased the need for social interaction. 
Second, the pandemics' impact on conceptual representations does not seem to be limited to abstract concepts. Instead, we also found an unusual pattern of results for more concrete concepts, that clustered mainly together, irrespectively of their sub-category. We found in the same cluster words referring to bodily parts, kinship, jobs, and places, along with words referred to diseases and lockdown-all words that held high Body-Object-Interaction and Imageability scores and low scores of Abstractness. This might be better interpreted when compared to Cluster 2, which features words that have higher scores of perceived Distance, lower scores in Confidence, and is situated in the bidimensional semantic space where Social Metacognition weights more. In this cluster, we found words directly related to the pandemics (Covid-19, herd immunity) or words that became related to the pandemics indirectly because their referents gained salient importance during the first lockdown (China).

Hence, the influence of Covid-19 seems to spread from the private to the public spheres, which interestingly correspond to Cluster 2 and 3 . The private sphere (Cluster 2) includes more concrete concepts and concepts referring to how the pandemic impacts our everyday life - two paradigmatic examples are lockdown and Italy. The public sphere (Cluster 3) includes more institutional terms and concepts directly referred to the pandemics, like epidemiological ones-two paradigmatic examples are State and herd immunity. Notably, this sphere is characterized by high scores in perceived Distance and associated with high uncertainty, as the low scores in Confidence testify.

How about the emotional, inner sphere? In such a sphere, mainly represented by Cluster 1, the influence of the pandemic is more indirect. Cluster 1 does not include terms directly related to Covid-19. However, ratings on emotionalinner and philosophical-spiritual concepts changed compared to previous databases, showing that we need others more to understand these concepts (higher scores in Social Metacognition). In addition, participants in our study had more difficulty in retrieving relevant contexts (lower scores of Contextual Availability), and in imagining (lower scores of Imageability) these kinds of concepts compared to the previous database.

In conclusion, our study has both theoretical and practical implications. On the theoretical side, it provides new insights into conceptual representation. First of all, our study shows that concreteness $\sim$ abstractness represents an important dimension to understand our conceptual organization. Second, it also emphasizes the role of affective, emotional, and interoceptive aspects for our concepts. Third, it highlights the crucial role of information on confidence to investigate abstract concepts-both in terms of inner monitoring and certainty of our own knowledge, and in terms of needing other people, experts or not, to complement our knowledge. Finally, it highlights our conceptual system's intrinsic flexibility, showing how the pandemic has profoundly and differently influenced our public, personal, and emotional spheres.

On a more practical side, understanding how people have changed their conception of different life spheres can offer hints that politicians, practitioners, and policymakers can exploit to address the pandemics. For example, the marked separation between the private and the public sphere that emerges from our results should be carefully considered for those who hope and plan a better society, where these two areas should be more integrated. As a further example, our results invite us to reflect on the role of science, perceived as an institution and delegated to the public sphere, rather than an instrument helpful for everyday citizen's life. Reflecting on the impact of Covid-19 on our concepts can thus contribute to understanding better what we want for our future.

Supplementary Information The online version contains supplementary material available at https://doi.org/10.1007/s00426-021-01633-z.

Author contributions All the authors contributed to develop and implement the idea of the study. IF and AHM prepared the questionnaires and collected the data. CM, IF, and AHM pre-processed and analyzed the data. CM and AMB drafted the paper, and all the other authors provided critical feedback. LT revised the paper and provided constructive comments. AHM provided helpful remarks on the final version of the text. All the authors have approved the final version of this manuscript.

Funding This study was funded by H2020-TRAINCREASE Project "From social interaction to abstract concepts and words: towards human centered technology development" CSA, Proposal n. 952324, P.I. Anna Borghi, and co-financed by the European Regional Development Fund (ERDF) and the Occitaine Region, convention number 182117SR. All data, scripts, and analyses are available at https://osf. io/h8t59/.

\section{Declarations}

Conflict of interest The authors declare no competing interest.

Ethical approval Ethics permission was granted by the Ethics Committee of the Department of Dynamic and Clinical Psychology, Sapienza University of Rome (Prot. n. 000275-23/03/2020). All participants provided informed consent.

\section{References}

Altarriba, J., Bauer, L. M., \& Benvenuto, C. (1999). Concreteness, context availability, and imageability ratings and word associations for abstract, concrete, and emotion words. Behavior Research Methods, Instruments, \&amp; Computers, 31, 578602. https://doi.org/10.3758/BF03200738

Barca, L., Burani, C., \& Arduino, L. S. (2002). Word naming times and psycholinguistic norms for Italian nouns. Behavior Research Methods, Instruments, \&amp; Computers, 34, 424434. https://doi.org/10.3758/BF03195471 
Barca, L., Mazzuca, C., \& Borghi, A. M. (2017). Pacifier overuse and conceptual relations of abstract and emotional concepts. Frontiers in Psychology. https://doi.org/10.3389/fpsyg.2017.02014

Barca, L., Mazzuca, C., \& Borghi, A. M. (2020). Overusing the Pacifier during Infancy Sets a Footprint on Abstract Words Processing. Journal of Child Language, 47(5), 1084-1099. https://doi.org/ 10.1017/S0305000920000070

Barsalou, L. W. (1993). Flexibility, structure, and linguistic vagary in concepts: Manifestations of a compositional system of perceptual symbols. In A. F. Collins, S. E. Gathercole, M. A. Conway, \& P. E. Morris (Eds.), Theories of memory (pp. 29-101). Lawrence Erlbaum Associates Inc.

Barsalou, L. W., Dutriaux, L., \& Scheepers, C. (2018). Moving beyond the distinction between concrete and abstract concepts. Philosophical Transactions of the Royal Society B: Biological Sciences, 373(1752), 20170144. https://doi.org/10.1098/rstb.2017.0144

Borghi, A. M., \& Binkofski, F. (2014). Words as social tools: An embodied view on abstract concepts. SpringerBriefs in Cognition series. Springer.

Borghi, A. M., Barca, L., Binkofski, F., \& Tummolini, L. (2018). Abstract concepts, language and sociality: From acquisition to inner speech. Philosophical Transactions of the Royal Society $b$ : Biological Sciences, 373(1752), 20170134.

Borghi, A. M., Barca, L., Binkofski, F., Castelfranchi, C., Pezzulo, G., \& Tummolini, L. (2019). Words as social tools: Language, sociality and inner grounding in abstract concepts. Physics of Life Reviews, 29, 120-153. https://doi.org/10.1016/j.plrev.2018.12.001

Borghi, A. M., Fini, C., \& Tummolini, L. (2021). Abstract concepts and metacognition: Searching for meaning in self and other. In M. D. Robinson \& L. E. Roberts (Eds.), Embodied psychology: Thinking, feeling, and acting. Springer

Borghi, A. M., Mazzuca, C., Da Rold, F., Falcinelli, I., Fini, C., Michalland, A. H., \& Tummolini, L. (2021). Abstract Words as Social Tools: Which Necessary Evidence? Frontiers in Psychology, 11, 3880 .

Chouikhi, H., Charrad, M., \& Ghazzali, N. (2015). A comparison study of clustering validity indices. In: 2015 Global Summit on Computer \& Information Technology (GSCIT), pp. 1-4. IEEE.

Cohen, J. (1988). Set correlation and contingency tables. Applied Psychological Measurement, 12(4), 425-434. https://doi.org/10.1177/ 014662168801200410

Connell, L. (2019). What have labels ever done for us? The linguistic shortcut in conceptual processing. Language, Cognition and Neuroscience, 34(10), 1308-1318.

Connell, L., \& Lynott, D. (2014). Principles of representation: Why you can't represent the same concept twice. Topics in Cognitive Science, 6, 390-406. https://doi.org/10.1111/tops.12097

Connell, L., Lynott, D., \& Banks, B. (2018). Interoception: The forgotten modality in perceptual grounding of abstract and concrete concepts. Philosophical Transaction of the Royal Society London b: Biological Science, 373(1752), 20170143.

Cuddy, A. J. C., et al. (2009). Stereotype content model across cultures: Towards universal similarities and some differences. British Journal of Social Psychology, 48(1), 1-33. https://doi.org/10.1348/ $014466608 X 314935$

Davis, M. H. (1980). A multidimensional approach to individual differences in empathy. JSAS Catalog of Selected Documents in Psychology, 10, 85.

Della Rosa, P. A., Catricalà, E., Vigliocco, G., \& Cappa, S. F. (2010). Beyond the abstract concrete dichotomy: Mode of acquisition, concreteness, imageability, familiarity, age of acquisition, context availability, and abstractness norms for a set of 417 Italian words. Behavior Research Methods, 42(4), 1042-1048. https://doi.org/ 10.3758/BRM.42.4.1042

Dove, G. (2018). Language as a disruptive technology: abstract concepts, embodiment and the flexible mind. Philosophical
Transaction of the Royal Society London B: Biological Science, 373(1752), 20170135.

Fini, C., \& Borghi, A. M. (2019). Sociality to reach objects and to catch meaning. Frontiers in Psychology, 10, 838. https://doi. org/10.3389/fpsyg.2019.00838

Gentner, D., \& Asmuth, J. (2019). Metaphoric extension, relational categories, and abstraction. Language, Cognition and Neuroscience, 34(10), 1298-1307.

Harpaintner, M., Trumpp, N. M., \& Kiefer, M. (2018). The semantic content of abstract concepts: A property listing study of 296 abstract words. Frontiers in Psychology, 9, 1748. https://doi. org/10.3389/fpsyg.2018.01748

Harrell, F. E. (2020). Hmisc: Harrell Miscellaneous. R package version 4.4-2; https://CRAN.R-project.org/package=Hmisc.

Jolliffe, I. T. (2010). Principal component analysis. Springer.

Kassambara, A., \& Mundt, F. (2019). Factoextra: Extract and Visualize the Results of Multivariate Data Analyses. R package version 1.0.6; https://CRAN.R-project.org/package=factoextra.

Kousta, S.-T., Vigliocco, G., Vinson, D. P., Andrews, M., \& Del Campo, E. (2011). The representation of abstract words: Why emotion matters. Journal of Experimental Psychology: General, 140(1), 14-34. https://doi.org/10.1037/a0021446

Kuhn, M., \& Wickham, H. (2020). Tidymodels: a collection of packages for modeling and machine learning using tidyverse principles; https://www.tidymodels.org.

Langland-Hassan, P., Faries, F. R., Gatyas, M., Dietz, A., \& Richardson, M. J. (2021). Assessing abstract thought and its relation to language with a new nonverbal paradigm: Evidence from aphasia. Cognition. https://doi.org/10.1016/j.cognition.2021.104622

Lê, S., Josse, J., \& Husson, F. (2008). FactoMineR: An R package for multivariate analysis. Journal of Statistical Software, 25(1), 1-18. hal-00359835.

Lenth, R. V. (2020). Emmeans: Estimated Marginal Means, aka Least-Squares Means. R package version: 1.5.3; https:// CRAN.R-project.org/package $=$ emmeans.

Lund, T. C., Sidhu, D. M., \& Pexman, P. M. (2019). Sensitivity to emotion information in children's lexical processing. Cognition, $190,61-71$.

Lupyan, G., \& Mirman, D. (2013). Linking language and categorization: evidence from aphasia. Cortex, 49(5), 1187-1194. https:// doi.org/10.1016/j.cortex.2012.06.006

Malt, B. C., \& Wolff, P. M. (2010). Words and the mind: How words capture human experience. Oxford University Press.

Mazzuca, C., Fini, C., Michalland, A. H., Falcinelli, I., Da Rold, F., Tummolini, L., \& Borghi, A. M. (2021a). From affordances to abstract words: The flexibility of sensorimotor grounding. Brain Sciences, 11(10), 1304.

Mazzuca, C., Falcinelli, I., Michalland, A. H., Tummolini, L., \& Borghi, A. M. (2021b). Differences and similarities in the conceptualization of COVID-19 and other diseases in the first Italian lockdown. Scientific Reports, 11(1), 1-12.

Mazzuca, C., Majid, A., Lugli, L., Nicoletti, R., \& Borghi, A. M. (2020). Gender is a multifaceted concept: evidence that specific life experiences differentially shape the concept of gender. Language and Cognition. https://doi.org/10.31219/osf.io/ugv43

McFalls, E. L., \& Schwanenflugel, P. J. (2002). The influence of contextual constraints on recall for words within sentences. The American Journal of Psychology, 115(1), 67-88. https://doi.org/ $10.2307 / 1423674$

Mehling, W. E., Price, C., Daubenmier, J. J., Acree, M., Bartmess, E., \& Stewart, A. (2012). The multidimensional assessment of interoceptive awareness (MAIA). PLoS ONE, 7(11), e48230. https://doi.org/10.1371/journal.pone.0048230

Murphy, G. L. (2002). The big book of concepts. A Bradford Book. Paivio, A. (1986). Mental representations. Oxford University Press. 
Paivio, A. (1990). Mental representations: A dual coding approach. Oxford University Press. https://doi.org/10.1093/acprof:oso/ 9780195066661.001.0001

Ponari, M., Norbury, C. F., \& Vigliocco, G. (2018). Acquisition of abstract concepts is influenced by emotional valence. Developmental Science, 21(2), e12549.

Rabb, N., Fernbach, P. M., \& Sloman, S. A. (2019). Individual representation in a community of knowledge. Trends in Cognitive Science, 23(10), 891-902.

Schwanenflugel, P. J., \& Shoben, E. J. (1983). Differential context effects in the comprehension of abstract and concrete verbal materials. Journal of Experimental Psychology: Learning, Memory, and Cognition, 9(1), 82-102. https://doi.org/10.1037/02787393.9.1.82

Shea, N. (2018). Metacognition and abstract concepts. Philosophical Transaction of the Royal Society London b: Biological Science, 373(1752), 20170133.

Singmann, H., Bolker, B., Westfall, J., Aust, F., \& Ben-Shachar, M. S. (2021). Afex:Analysis of Factorial Experiments. R package version 0.28-1; https://CRAN.R-project.org/package=afex.

Spitzer, R. L., Kroenke, K., Williams, J. B., \& Löwe, B. (2006). A brief measure for assessing generalized anxiety disorder: the GAD-7. Archives of Internal Medicine, 166(10), 1092-1097. https://doi. org/10.1001/archinte.166.10.1092
Thompson, B., Roberts, S. G., \& Lupyan, G. (2020). Cultural influences on word meanings revealed through large-scale semantic alignment. Nature Human Behaviour, 4(10), 1029-1038.

Tillotson, S. M., Siakaluk, P. D., \& Pexman, P. M. (2008). Bodyobject interaction ratings for 1,618 monosyllabic nouns. Behavior Research Methods, 40(4), 1075-1078. https://doi.org/10.3758/ BRM.40.4.1075

Villani, C., Lugli, L., Liuzza, M., \& Borghi, A. M. (2019). Varieties of abstract concepts and their multiple dimensions. Language and Cognition, 11(3), 403-430. https://doi.org/10.1017/langcog.2019. 23

Villani, C., Lugli, L., Liuzza, M. T., Nicoletti, R., \& Borghi, A. M. (2021). Sensorimotor and interoceptive dimensions in concrete and abstract concepts. Journal of Memory and Language, 116, 104173. https://doi.org/10.17605/OSF.IO/YPX7S

Wei, T., \& Simko, V. (2017). R package "corrplot": Visualization of a Correlation Matrix (Version 0.84). https://github.com/taiyun/ corrplot.

Publisher's Note Springer Nature remains neutral with regard to jurisdictional claims in published maps and institutional affiliations. 